\title{
First assimilations of COSMIC radio occultation data into the Electron Density Assimilative Model (EDAM)
}

\author{
M. J. Angling \\ QinetiQ, St. Andrew's Road, Malvern, Worcs, WR14 3PS, UK \\ Received: 15 January 2007 - Revised: 27 June 2007 - Accepted: 28 June 2007 - Published: 26 February 2008
}

\begin{abstract}
Ground based measurements of slant total electron content (TEC) can be assimilated into ionospheric models to produce 3-D representations of ionospheric electron density. The Electron Density Assimilative Model (EDAM) has been developed for this purpose. Previous tests using EDAM and ground based data have demonstrated that the information on the vertical structure of the ionosphere is limited in this type of data. The launch of the COSMIC satellite constellation provides the opportunity to use radio occultation data which has more vertical information. EDAM assimilations have been run for three time periods representing quiet, moderate and disturbed geomagnetic conditions. For each run, three data sets have been ingested - only ground based data, only COSMIC data and both ground based and COSMIC data. The results from this preliminary study show that both ground and space based data are capable of improving the representation of the vertical structure of the ionosphere. However, the analysis is limited by the incomplete deployment of the COSMIC constellation and the use of auto-scaled ionosonde data. The first of these can be addressed by repeating this type of study once full deployment has been achieved. The latter requires the manual scaling of ionosonde data; ideally an agreed data set would be scaled and made available to the community to facilitate comparative testing of assimilative models.
\end{abstract}

Keywords. Ionosphere (Modeling and forecasting; Instruments and techniques) - Radio science (Ionospheric propagation)

\section{Introduction}

Comprehensive, global and timely specifications of the earth's atmosphere (particularly refractivity profiles of the

Correspondence to: $\mathrm{M}$. J. Angling

(mjangling@qinetiq.com) troposphere and ionosphere) are required to ensure the effective operation, planning and management of many radio frequency systems. One way of providing ionospheric refractivity information is to employ an ionospheric data assimilation system. Such systems can produce 3-D images of the ionosphere using data provided by a range of measurement techniques. Of particular note is data from the International GNSS Service (IGS) receiver network (Beutler et al., 1999). Many IGS stations provide data in hourly files with low latency (i.e. files become available within $\sim 30 \mathrm{~min}$ of the data being collected), which can be used to calculate slant total electron content (TEC).

Radio occultation (RO) methods are also being increasingly investigated. RO measurements are made by monitoring transmissions from GPS satellites using receivers on low earth orbiting (LEO) satellites and provide the potential of measuring refractivity profiles in regions where ground based sensors cannot easily be located, such as deep sea waters. The recent launch of RO instruments on the Constellation Observing System for Meteorology, Ionosphere \& Climate (COSMIC) six satellite constellation has the potential to provide a large amount of ionospheric data (Hajj et al., 2000); again this will be available with a low latency $(115 \mathrm{~min})$. For both ground and space based data, data assimilation provides an optimal way of combining slant TEC measurements with an ionospheric model to provide a full 3-D representation of the ionospheric electron density.

The Electron Density Assimilative Model (EDAM) has been developed (Angling and Cannon, 2004; Angling and Khattatov, 2006) to assimilate measurements into a background ionospheric model. This model is provided by PIM (Daniell et al., 1995) and the majority of the input data is GPS TEC derived from IGS stations. The assimilation is based on a weighted, damped least mean squares estimation. This is a form of minimum variance optimal estimation (also referred to as best linear unbiased estimation, BLUE) that provides an expression for an updated estimation of the state (known as

Published by Copernicus Publications on behalf of the European Geosciences Union. 


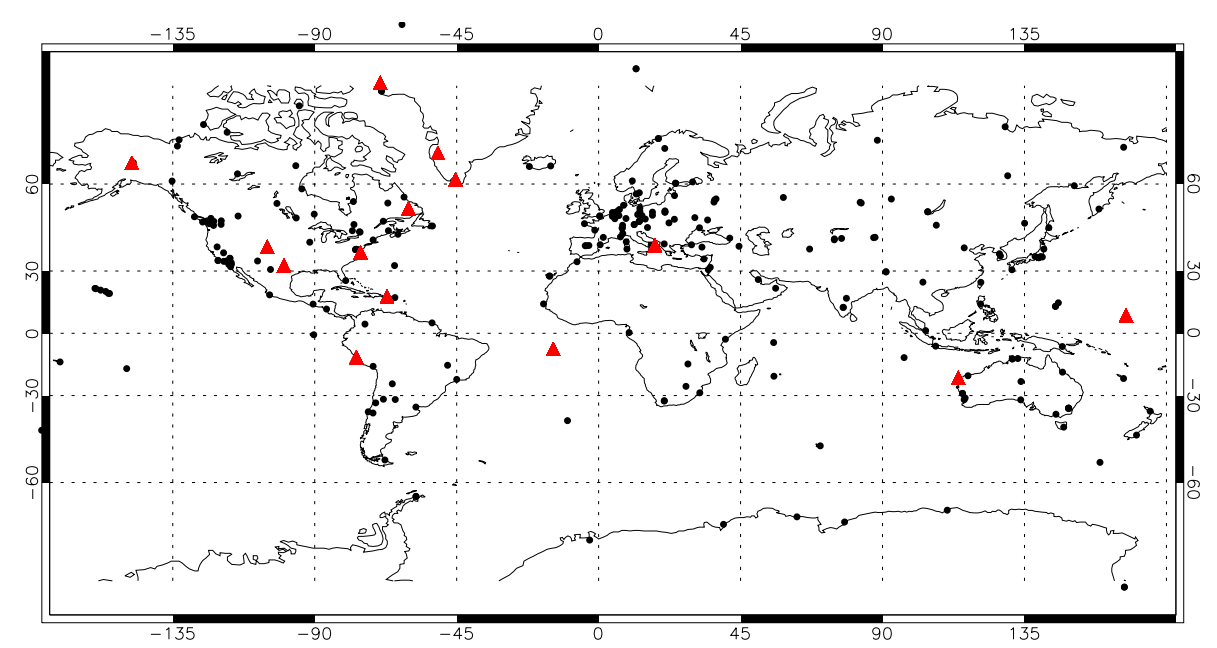

Fig. 1. Map showing locations of IGS stations (dots) and vertical ionosondes (triangles).

the analysis) that is dependent upon an initial estimate of the state (the background model), and the differences between the background model and the observations (Menke, 1989). The error covariance matrices of the background model and the observations are also included to control the relative contributions of the background and the observations to the analysis:

$\boldsymbol{x}_{a}=\boldsymbol{x}_{b}+\mathbf{K}\left(\mathbf{y}-\mathbf{H} \boldsymbol{x}_{b}\right)$

$\mathbf{K}=\mathbf{B H}^{T}\left(\mathbf{H B H}^{T}+\mathbf{R}\right)^{-\mathbf{1}}$

where $\boldsymbol{x}_{a}$ is the analysis, $\boldsymbol{x}_{b}$ is the background model, $\mathbf{K}$ is the weight matrix, $\boldsymbol{y}$ is the observation vector, $\mathbf{B}$ is the background error covariance matrix, and $\mathbf{R}$ is the error covariance matrix of the observations (Rodgers, 2000). $\mathbf{H}$ is the observation operator that relates the measurements to the state:

$\boldsymbol{y}=\mathbf{H x}+\varepsilon$

where $\varepsilon$ is the observation error. The analysis error covariance matrix $(\mathbf{S})$ may also be calculated thus:

$$
\begin{aligned}
& \mathbf{S}=\mathbf{B}-\mathbf{B H}^{T}\left(\mathbf{H B H}^{T}+\mathbf{R}\right)^{-1} \mathbf{H B} \\
& \mathbf{S}=\mathbf{B}-\mathbf{K H B}
\end{aligned}
$$

The assimilation is conducted using a magnetic coordinate system that remains fixed in space with respect to the sun. An assimilation time step of $15 \mathrm{~min}$ has been used and the electron density differences between the voxels of the analysis and the background model are propagated from one time step to the next by assuming persistence combined with an exponential decay. The time constant for this decay is set at $4 \mathrm{~h}$. Thus if the data feed is interrupted, the analysis will decay back to the background model.

Previous testing (Angling and Khattatov, 2006) has demonstrated that it is difficult to modify the vertical structure of the EDAM electron density grid using ground based
TEC data alone - this is a problem common to all assimilative approaches. It is anticipated that the introduction of RO data will provide much better vertical information and, therefore, improve the vertical representation of the ionosphere. The following section describes the first tests of EDAM using both ground based IGS data and space based data from COSMIC.

\section{Test scenarios}

Three test periods have been used for this study. These are 19 to 20 August 2006 and 4 and 10 September 2006. These periods correspond to disturbed, moderate and quiet geomagnetic conditions, respectively. For each period, three assimilation runs have been conducted using different input data: assimilation of just RO data, assimilation of just ground based GPS TEC data, and assimilation of both RO and ground based TEC.

The ground based data has been obtained from the 277 IGS sites shown in Fig. 1. Where available, differential code biases (DCBs) from the Centre for Orbit Determination in Europe (CODE) are used to provide calibrated TEC. Otherwise, the station DCB is determined by EDAM itself as part of the assimilation process. The IGS sites record data with a period of $30 \mathrm{~s}$. This data is then averaged to reduce the sampling to a $5 \mathrm{~min}$ period. Thus, given 277 IGS sites, and assuming that each is locked to eight satellites, approximately 6500 slant TEC measurements will be assimilated within one 15 min assimilation period.

The COSMIC data has been provided by the COSMIC Data Analysis and Archive Centre (CDAAC) and takes the form of calibrated TEC from the COSMIC satellites' precise orbit determination (pod) antenna. Consequently, the dataset contains measurements made with both a true RO geometry as well as topside measurements to GPS satellites at high 


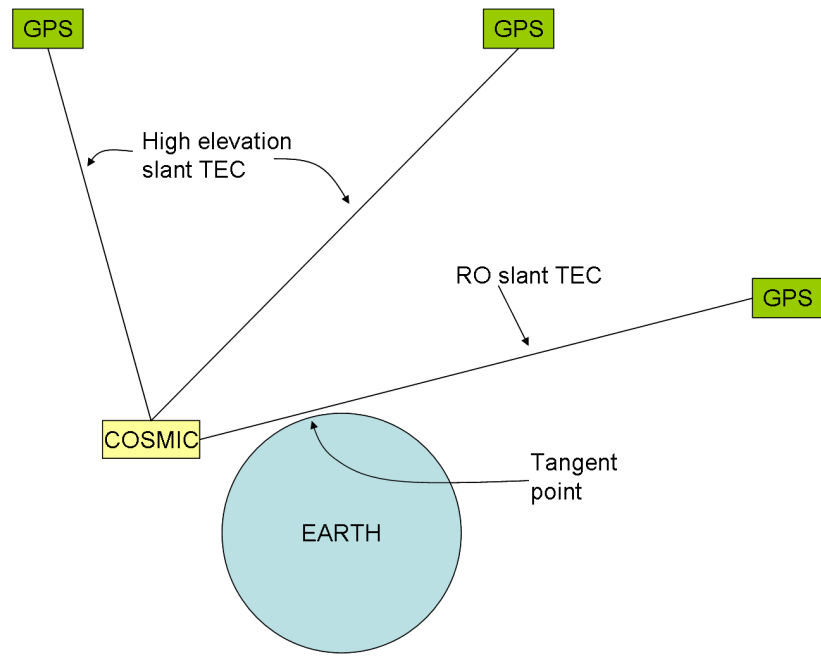

Fig. 2. Schematic diagram of COSMIC observation geometry.

elevations with respect to the GPS receiver (Fig. 2). Both types of measurements are assimilated. The full sampling rate of $1 \mathrm{~Hz}$ is used for the $\mathrm{RO}$ measurements, whilst the sampling rate is reduced (to a minimum of $0.01 \mathrm{~Hz}$ at zenith) for the high elevation COSMIC TEC data to reduce the computation times of the assimilation.

Again, if it is assumed that each COSMIC receiver is locked to eight high elevation GPS satellites, then approximately 400 high elevation slant TECs will be assimilated within one $15 \mathrm{~min}$ assimilation period. For the periods under consideration, COSMIC made approximately $2000 \mathrm{RO}$ measurements per day. Therefore, approximately $20 \mathrm{RO}$ measurements will be assimilated during each $15 \mathrm{~min}$ assimilation period. It should also be noted that, since the COSMIC constellation was not fully deployed at the times of the tests, the measurements tend to be spatially clustered.

\section{Results}

3.1 Validation using vertical ionosonde electron density profiles

The EDAM results have first been compared to electron density profiles derived from vertical ionosonde measurements of the ionosphere. In particular data from the USAF Digital Ionospheric Sounding System (DISS) network (shown as triangles in Fig. 1) has been used. It should be noted that the ionosonde data is autoscaled and consequently the vertical profiles may contain errors. Furthermore, the topside electron density profiles above the peak of the $\mathrm{F}$ region have been constructed using the Chapman scale height $H_{T}$ at the F2-layer peak (Reinisch and Huang, 2001; Reinisch et al., 2004). To make the comparison vertical profiles have been extracted from the EDAM electron density grid at the location of each ionosonde and both the EDAM and ionosonde

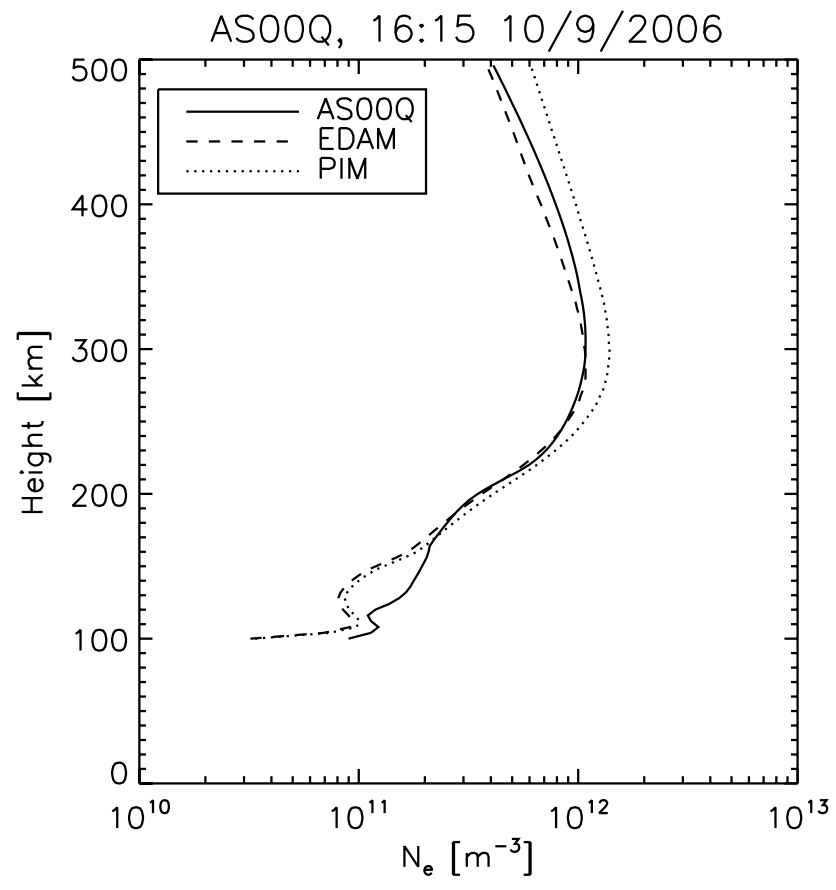

Fig. 3. Example comparison between PIM, EDAM and a vertical electron density profile measured by the Ascension Island ionosonde.

vertical profiles are interpolated onto $5 \mathrm{~km}$ vertical steps. No attempt has been made to limit the validation data to that which lies close (in space or time) to the COSMIC or IGS data ingested by EDAM. An example comparison between PIM, EDAM and a vertical profile from Ascension Island $\left(7.9^{\circ} \mathrm{S}, 345.6^{\circ} \mathrm{E}\right)$ is shown in Fig. 3.

Root mean square (RMS) differences have been calculated between the vertical ionosonde profiles and the EDAM profiles at each $5 \mathrm{~km}$ height step. No quality control has been applied to the ionosonde profiles beyond the requirement that the electron density must be positive. Consequently any spurious results have been included. The results for 10 and 4 September (1252 and 1306 profiles, respectively) and for 19 20 August (2522 profiles) are given in Figs. 4 to 6, respectively. In each case the RMS error of PIM with respect to the ionosonde vertical profile is shown by the solid line, the RMS error of EDAM with only IGS input is shown by the dashed line, the RMS error of EDAM with only RO input is shown by the dotted line, and the RMS error of EDAM with IGS and RO input is shown by the dash-dot line.

Figure 4 shows that for quiet geomagnetic conditions, the assimilation of IGS and RO data makes little difference to the RMS error. For moderate conditions (Fig. 5), EDAM improves the RMS error at all heights above $200 \mathrm{~km}$ when assimilating the COSMIC data. When using only IGS data the greatest gains occur at approximately $250 \mathrm{~km}$, but performance degrades to a similar level as PIM by $350 \mathrm{~km}$. When both IGS and COSMIC data are assimilated, the results show 


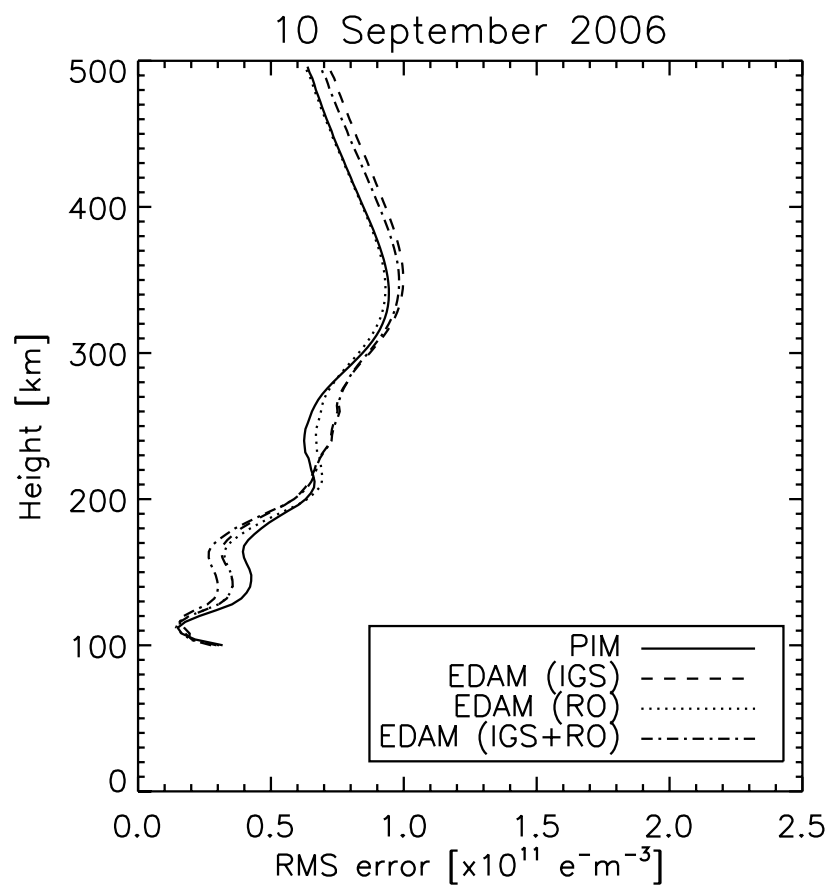

Fig. 4. RMS error between ionospheric models and ionosonde vertical profiles at $5 \mathrm{~km}$ height steps on 10 September 2006 (quiet geomagnetic conditions). The models are: PIM (solid line); EDAM assimilating IGS data only (dashed line); EDAM assimilating COSMIC RO data only (dotted line); and EDAM assimilating IGS and COSMIC RO data (dot-dash line).

an improvement over the purely IGS case between approximately 270 and $380 \mathrm{~km}$. However, it is clear that this assimilation does not provide an ideal combination of the two types of data since the combined RMS error is only the lowest of the tests over a small vertical range from 270 to $320 \mathrm{~km}$. For the disturbed test (19-20 August, Fig. 6), the COSMIC data assimilation provides the best results, again improving RMS error at all heights above $200 \mathrm{~km}$. However, when IGS data is assimilated, EDAM exhibits degraded RMS performance in comparison to PIM at heights around the F region peak. This is due to mis-modelling of the vertical structure when the ground based data is used. The combined IGS/COSMIC assimilation shows some improvement, but remains worse than PIM around the F region peak. This indicates that the assimilation results are being dominated by the ground based measurements.

\subsection{Validation using Abel Transform vertical profiles}

The EDAM results have also been compared to vertical profiles derived from the COSMIC RO measurements using an Abel Transform (Fjeldbo et al., 1971; Hajj and Romans, 1998). The latter were obtained from CDAAC. Since the same input slant TEC data used in the Abel Transform has been assimilated in EDAM, the Abel Transform profiles do

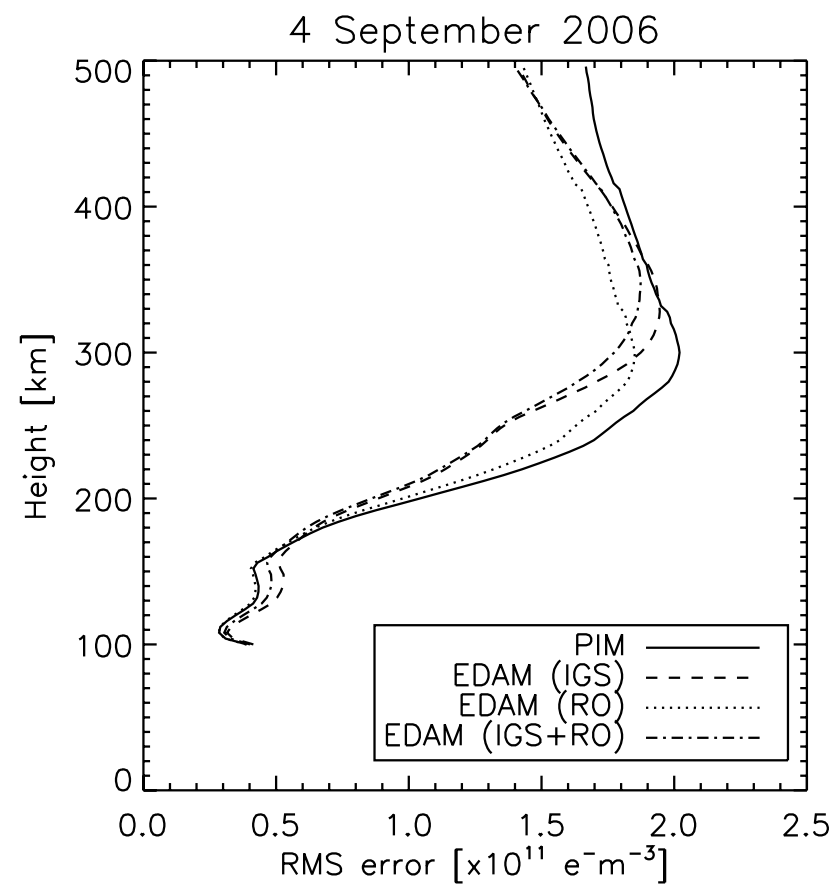

Fig. 5. RMS error between ionospheric models and ionosonde vertical profiles at $5 \mathrm{~km}$ height steps on 4 September 2006 (moderate geomagnetic conditions). The models are: PIM (solid line); EDAM assimilating IGS data only (dashed line); EDAM assimilating COSMIC RO data only (dotted line); and EDAM assimilating IGS and COSMIC RO data (dot-dash line).

not provide a truly independent test of the assimilation. It should also be remembered that the two profiles actually represent different electron density fields - the EDAM profile is an estimation of the vertical profile at a particular location, whilst the Abel Transform profile is, in some sense, an average representation of the ionosphere over the horizontal extent of the measurement.

To make the comparison a vertical profile has been extracted from the EDAM electron density grid at the location of the RO tangent point half way through the measurement period. The tangent point is the location where the ray from the GPS transmitter to the COSMIC receiver makes its closest approach to the Earth's surface (Fig. 2). The tangent point half way through the measurement period is used because the tangent point drifts during the measurement due to the non-ideal measurement geometry. Both the EDAM and Abel Transform vertical profiles are interpolated onto $5 \mathrm{~km}$ vertical steps. An example comparison is shown in Fig. 7.

Root mean square (RMS) differences have been calculated between the Abel Transform profile and the EDAM profiles at each $5 \mathrm{~km}$ height step. No quality control has been applied to the Abel Transform profiles beyond the requirement that the electron density must be positive. Consequently any spurious results, such as those below $200 \mathrm{~km}$ in Fig. 7, have been included. The results for 10 and 4 September (2066 and 1861 


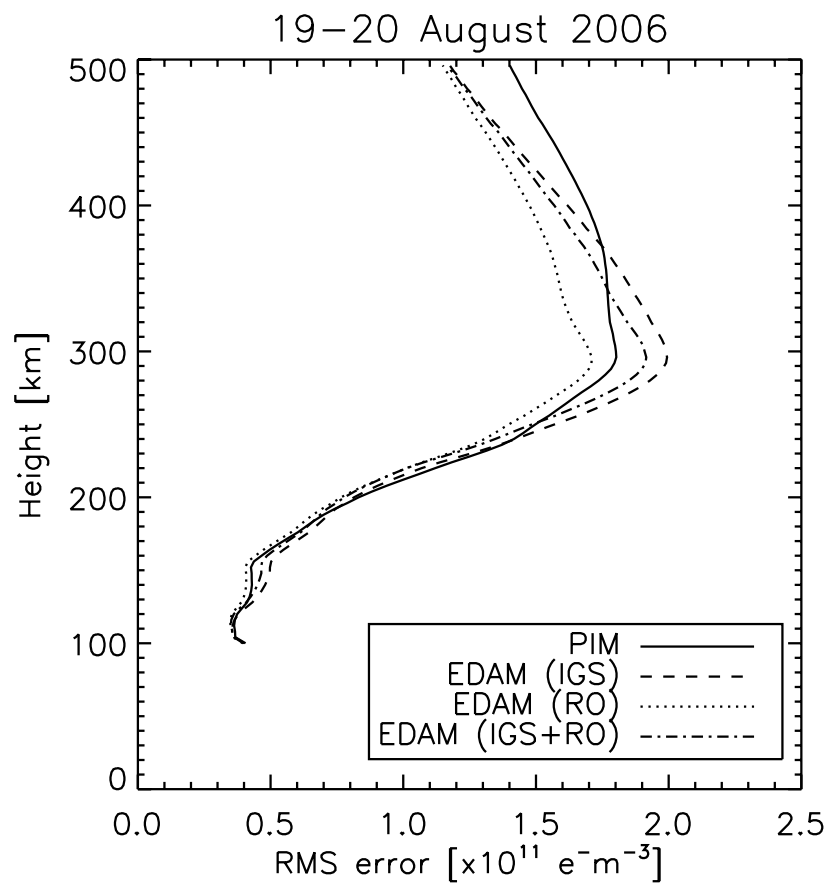

Fig. 6. RMS error between ionospheric models and ionosonde vertical profiles at $5 \mathrm{~km}$ height steps on 19-20 August 2006 (disturbed geomagnetic conditions). The models are: PIM (solid line); EDAM assimilating IGS data only (dashed line); EDAM assimilating COSMIC RO data only (dotted line); and EDAM assimilating IGS and COSMIC RO data (dot-dash line).

profiles, respectively) and for 19-20 August (4184 profiles) are given in Figs. 8 to 10, respectively. In each case the RMS error of PIM with respect to the Abel Transform vertical profile is shown by the solid line, the RMS error of EDAM with only IGS input is shown by the dashed line, the RMS error of EDAM with only RO input is shown by the dotted line, and the RMS error of EDAM with IGS and RO input is shown by the dash-dot line.

Figure 8 shows that for quiet geomagnetic conditions, the assimilation of IGS and RO data makes little difference to the RMS errors - though some improvement can be seen above $300 \mathrm{~km}$. However, for moderate and disturbed conditions (Figs. 9 and 10), EDAM generally performs better than PIM. As expected, the assimilation of the RO slant TEC data has the greatest impact on the assimilation results, though the improved RMS exhibited by EDAM ingesting just ground based IGS data does demonstrate that it is possible to reduce errors in the vertical structure of the ionospheric model using this data.

\section{Discussion}

The use of the Abel Transform vertical profiles to validate EDAM assimilations that have used COSMIC data is not

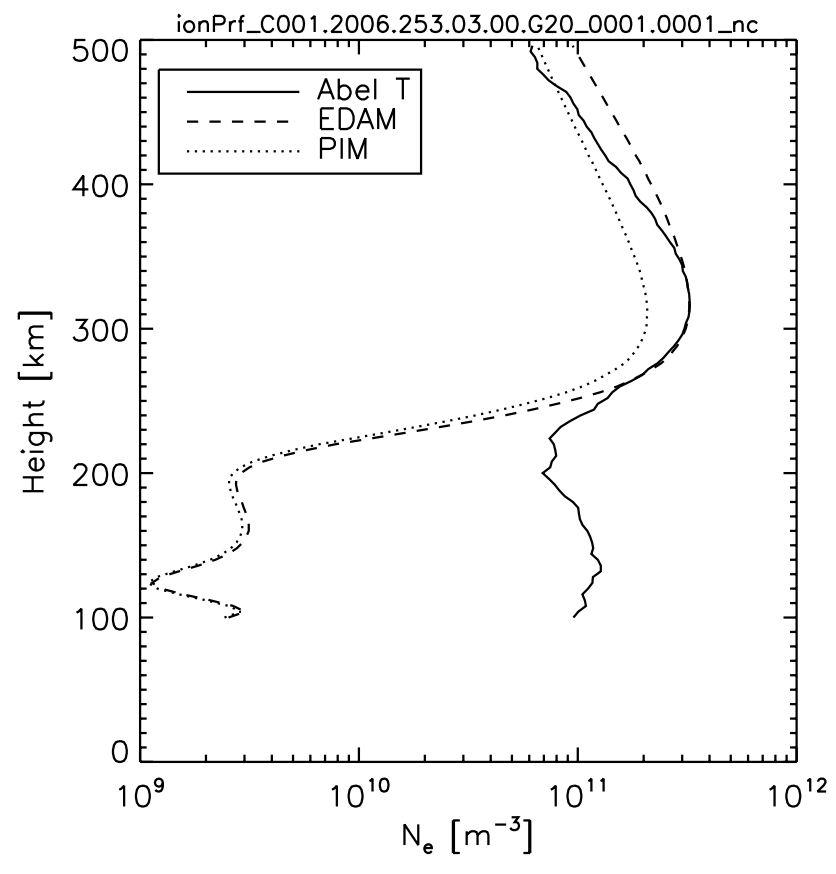

Fig. 7. Example comparison between PIM, EDAM and Abel Transform vertical electron density profiles.

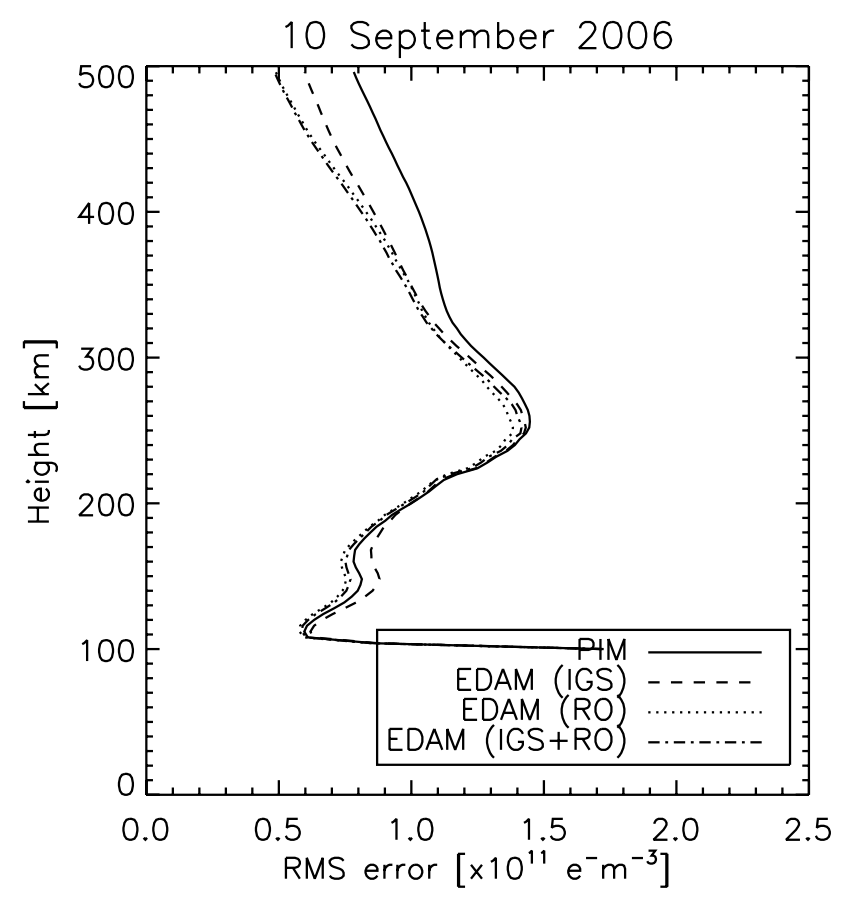

Fig. 8. RMS error between ionospheric models and Abel transform vertical profiles at $5 \mathrm{~km}$ height steps on 10 September 2006 (quiet geomagnetic conditions). The models are: PIM (solid line); EDAM assimilating IGS data only (dashed line); EDAM assimilating COSMIC RO data only (dotted line); and EDAM assimilating IGS and COSMIC RO data (dot-dash line). 


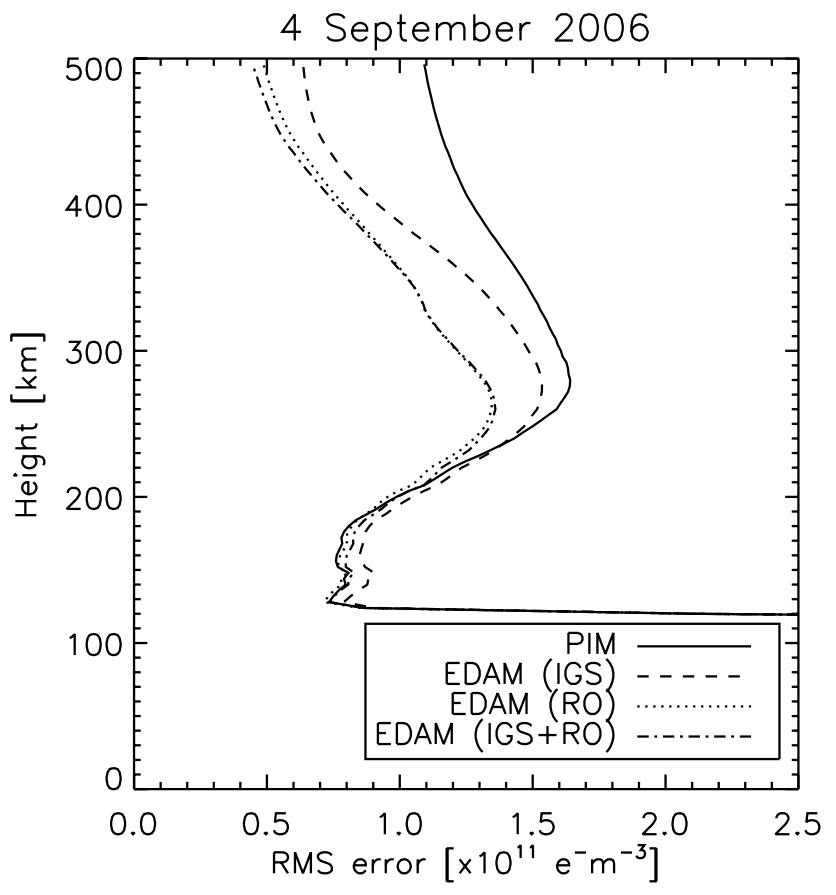

Fig. 9. RMS error between ionospheric models and Abel transform vertical profiles at $5 \mathrm{~km}$ height steps on 4 September 2006 (moderate geomagnetic conditions). The models are: PIM (solid line); EDAM assimilating IGS data only (dashed line); EDAM assimilating COSMIC RO data only (dotted line); and EDAM assimilating IGS and COSMIC RO data (dot-dash line).

entirely legitimate since the profiles are not truly independent. The impact of this can be seen in the close agreement between the results for assimilations using only COSMIC data and those using both IGS and COSMIC data. The results are clearly dominated by the COSMIC data. However, in the case of assimilating IGS data alone, the vertical profiles are independent. The RMS error reduction is not as great, but the results do show that it is possible to improve the representation of the vertical structure of the ionosphere using only ground based sensors.

Profiles from vertical ionosondes do provide independent validation data, though, in this study, the accuracy is limited by the auto-scaling. Furthermore, for a ground based sounder, the topside ionosphere is necessarily reconstructed by means of a model based on scale heights measured near the F region peak. Results published by Belehaki and Kersley (2006) indicate that the reconstruction tends to result in a positive electron density bias above the F region peak. In the daytime, this reduces with height to become a small negative bias at higher altitudes. The EDAM RMS results indicate that, for this test, the results are dominated by the ground based IGS data to the extent that the combined assimilation results (i.e. IGS and COSMIC data) closely follow the IGS alone results. Indeed, the addition of the IGS data can be seen to degrade EDAM's performance; this is especially ev-

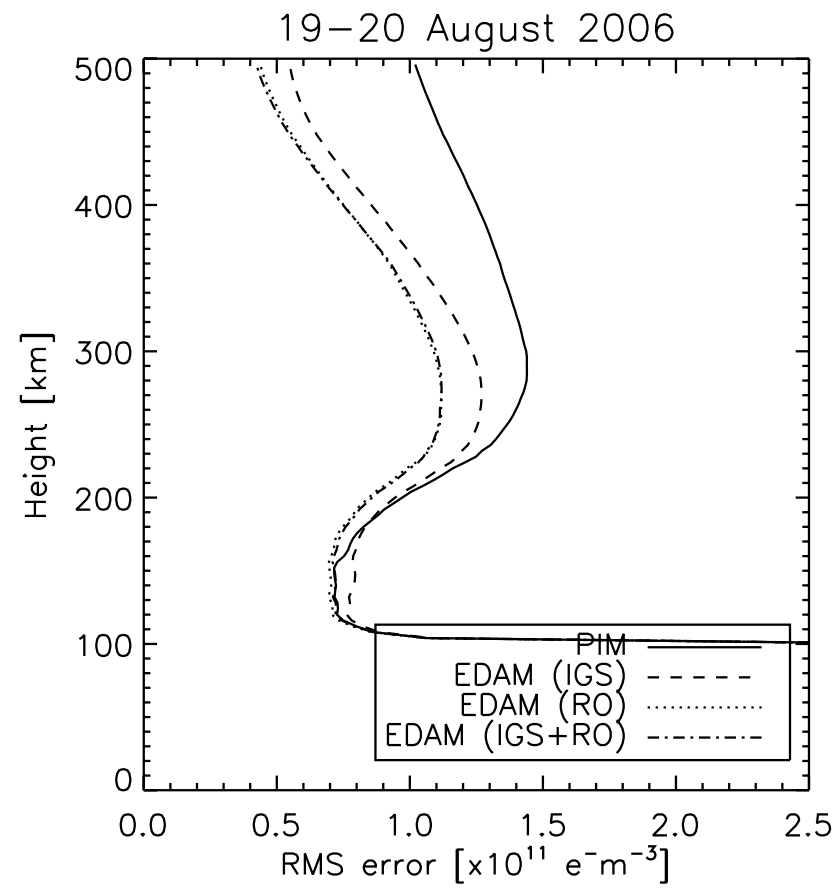

Fig. 10. RMS error between ionospheric models and Abel transform vertical profiles at $5 \mathrm{~km}$ height steps on 19-20 August 2006 (disturbed geomagnetic conditions). The models are: PIM (solid line); EDAM assimilating IGS data only (dashed line); EDAM assimilating COSMIC RO data only (dotted line); and EDAM assimilating IGS and COSMIC RO data (dot-dash line).

ident in the disturbed test where the degradation occurs over most of the height range and results in errors in excess of those displayed by the background model (PIM). It is likely that the performance degradation is due to mis-modelling of the vertical structure of the ionosphere, but it is not clear why this problem is apparent in the vertical ionosonde tests and not in the Abel Transform tests.

In these tests the improvements shown by EDAM in comparison to PIM are rather modest. This can be attributed to three reasons: first and most fundamental is that the chosen method of testing provides a very stringent test of the ionospheric vertical structure as modelled by EDAM, such that small errors in profile heights can result in large electron density errors. Secondly, no quality control has been applied to the vertical electron density profiles used for validation. This is especially a problem in the case of the vertical ionosondes as auto-scaled data has been used. And thirdly, the COSMIC constellation was not fully deployed at the time of the test, leading to clustering of the measurements. Consequently, the impact of the final constellation on global assimilations cannot be assessed. 


\section{Conclusions}

EDAM assimilations have been run for three time periods representing quiet, moderate and disturbed geomagnetic conditions. For each run, three data sets have been ingested only IGS data, only COSMIC data and both IGS and COSMIC data. The results show that both ground and space based data are capable of improving the representation of the vertical structure of the ionosphere, However, the analysis is limited by the incomplete deployment of the COSMIC constellation and the use of auto-scaled ionosonde data. The first of these can be addressed by repeating this type of study once full deployment has been achieved. The latter requires the manual scaling of ionosonde data; ideally an agreed data set would be scaled and made available to the community to facilitate comparative testing of assimilative models.

Acknowledgements. EDAM has been developed under funding from the Operating Environment domain of the United Kingdom Ministry of Defence Corporate Science and Technology programme. IGS data was obtained from the IGS JPL and SOPAC Data Centres. Differential code biases were obtained from the Centre for Orbit Determination in Europe. Vertical ionosonde data was provided by the U.S. Air Force Research Laboratory. COSMIC data was provided by the Taiwan National Space Organization (NSPO) and the U.S. University Corporation for Atmospheric Research (UCAR) via the COSMIC Data Analysis and Archive Centre.

Topical Editor U.-P. Hoppe thanks L. McNamara and S. E. Pryse for their help in evaluating this paper.

\section{References}

Angling, M. J. and Cannon, P. S.: Assimilation of radio occultation measurements into background ionospheric models, Radio Sci., 39, RS1S08, doi:10.1029/2002RS002819, 2004.
Angling, M. J. and Khattatov, B.: Comparative study of two assimilative models of the ionosphere, Radio Sci., 41, RS5S20, doi:10.1029/2005RS003372, 2006.

Belehaki, A. and Kersley, L.: Statistical validation of a technique for estimating total electron content from bottomside ionospheric profiles, Radio Sci., 41, RS5003, doi:10.1029/2005RS003433, 2006.

Beutler, G., Rothacher, M., Schaer, S., Springer, T. A., Kouba, J., and Neilan, R. E.: The International GPS Service (IGS): An Interdisciplinary Service in Support of Earth Sciences, Adv. Space Res., 23(4), 631-635, 1999.

Daniell Jr., R. E., Brown, L. D., Anderson, D. N., Fox, M. W., Doherty, P. H., Decker, D. T., Sojka, J. J., and Schunk, R. W.: Parameterized ionospheric model: A global ionospheric parameterization based on first principles models, Radio Sci., 30(5), 1499-1510, doi:10.1029/95RS01826, 1995.

Fjeldbo, G., Kliore, A. J., and Eshleman, R.: The neutral atmosphere of Venus as studied with the Mariner V radio occultation experiment, The Astronomical Journal, 76(2), 123-141, 1971.

Hajj, G. A., Lee, L. C., Pi, X., Romans, L. J., Schreiner, W. S., Straus, P. R., and Wang, C.: COSMIC GPS Ionospheric Sensing and Space Weather, Special Issue of Terrestrial Atmos. Oceanic Sci., 11, 235-272, 2000.

Hajj, G. A. and Romans, L. J.: Ionospheric electron density profiles obtained with the Global Positioning System: Results from the GPS/MET experiment, Radio Sci., 33, 175-190, 1998.

Menke, W.: Geophysical data analysis: discrete inverse theory, International Geophysics Series, San Diego, Academic Press, 1989.

Reinisch, B. W. and Huang, X.: Deducing topside profiles and total electron content from bottomside ionograms, Adv. Space Res., 27(1), 23-30, 2001.

Reinisch, B. W., Huang, X., Belehaki, A., and Ilma, R.: Using scale heights derived from bottomside ionograms for modelling the IRI topside profile, Adv. Radio Sci., 2, 293-297, 2004, http://www.adv-radio-sci.net/2/293/2004/.

Rodgers, C. D.: Inverse methods for atmospheric sounding: theory and practice, Series on Atmospheric, Ocean. Planet. Phys., 2, World Scientific Publishing, 2000. 